\title{
Optical spectroscopy and current detection during warm-up and destruction of impurity-helium condensates
}

\author{
I.N. Krushinskaya ${ }^{1}$, R.E. Boltnev ${ }^{1,2}$, I.B. Bykhalo ${ }^{1}$, A.A. Pelmenev ${ }^{1,2}$, \\ V.V. Khmelenko ${ }^{3}$, and D.M. Lee ${ }^{3}$ \\ ${ }^{I}$ Branch of Talroze Institute for Energy Problems of Chemical Physics, \\ Russian Academy of Sciences, Chernogolovka 142432, Moscow Region, Russia \\ E-mail: pelmenev@binep.ac.ru \\ ${ }^{2}$ Joint Institute for High Temperatures, Russian Academy of Sciences, Moscow 125412, Russia \\ ${ }^{3}$ Institute for Quantum Science and Engineering, Department of Physics and Astronomy, \\ Texas A\&M University, College Station, Texas 77843, USA
}

Received October 30, 2014, published online April 23, 2015

\begin{abstract}
New experimental results on detection of optical spectra and ion currents during destruction of impurity-helium condensates (IHCs) have been obtained. It is shown that emission during IHC sample destruction is accompanied by current pulses, pressure peaks and temperature changes. The molecular bands of excimer molecules $\mathrm{XeO}^{*}$ are assigned to molecules stabilized in films of molecular nitrogen covering the heavier cores of impurity clusters which form impurity-helium condensates.

PACS: $76.30 . \mathrm{Rn}$ Free radicals;

78.60.-b Other luminescence and radiative recombination;

61.46.-w Structure of nanoscale materials.
\end{abstract}

Keywords: free radicals, optical spectroscopy, condensed helium, nanoclusters.

\section{Introduction}

It is well known that deposition of rare gases (RG) passed through an electrical discharge onto a cold $(\sim 4 \mathrm{~K})$ surface, or irradiation of cryofilms by energetic particles (electrons, protons, or photons with energies of $20 \mathrm{eV}-4 \mathrm{MeV}$ ) can cause formation and stabilization of neutral radicals and ions [1-7].

Recent experiments [8] have revealed ion currents accompanied by luminescence during destruction of nitrogenhelium condensates prepared by condensation of nitrogenhelium gas mixtures (after passing through a radio-frequency (rf) discharge zone) into bulk superfluid helium (He II). We present new experimental results on the detection of optical spectra and ion currents during thermostimulated destruction of impurity-helium condensates (IHCs) prepared from nitrogen-argon-helium and nitrogen-xenon-helium gas mixtures.

\section{Experimental setup}

The experimental technique of IHC sample preparation was first developed in 1974 [9]. A cryogenic portion of the experimental setup is shown in Fig. 1. It is based on the injection of a helium gas jet containing impurity particles
( Im $=\mathrm{N}, \mathrm{N}_{2}, \mathrm{H}, \mathrm{H}_{2}, \mathrm{Ne}, \mathrm{Ar}, \mathrm{Kr}$, etc.) into bulk He II. A gas mixture enters a helium bath region from a quartz capillary cooled with liquid nitrogen inside an atom source as designated by position 3 (pos. 3) in Fig. 1(a). The lower portion of the capillary is surrounded by electrodes (pos. 2 in Fig. 1(a)) to produce an $\mathrm{rf}$ discharge $(f=40-52 \mathrm{MHz}, P=$ $=40-90 \mathrm{~W})$. The typical conditions during sample preparation were as follows: the impurity admixture, $[\mathrm{Im}] /[\mathrm{He}] \sim 0.5-1 \%$, the gas jet flux $(4.5-6) \cdot 10^{19} \mathrm{~s}^{-1}$, the superfluid helium temperature $1.5 \mathrm{~K}$, and the duration of the sample condensation 600-5000 s. Upon fast cooling of the gas jet, impurity nanoclusters with characteristic size of $5-6 \mathrm{~nm}$ form in the gas phase $[10,11]$. In bulk He II the nanoclusters aggregate into porous condensates with impurity particle densities $\sim 10^{19}-10^{20} \mathrm{~cm}^{-3}[10-12]$.

The oxygen content in the gas mixtures is mainly a result of contamination of the helium gas. We employ helium gas with an oxygen content of $\sim 10 \mathrm{ppm}$. A gas jet (pos. 5 in Fig. 1(a)) consisting of a mixture of helium and impurity gases was directed onto the surface of superfluid helium contained in a glass beaker (pos. 6 on Fig. 1(a)) placed below the source at a distance of $20-35 \mathrm{~mm}$. The 


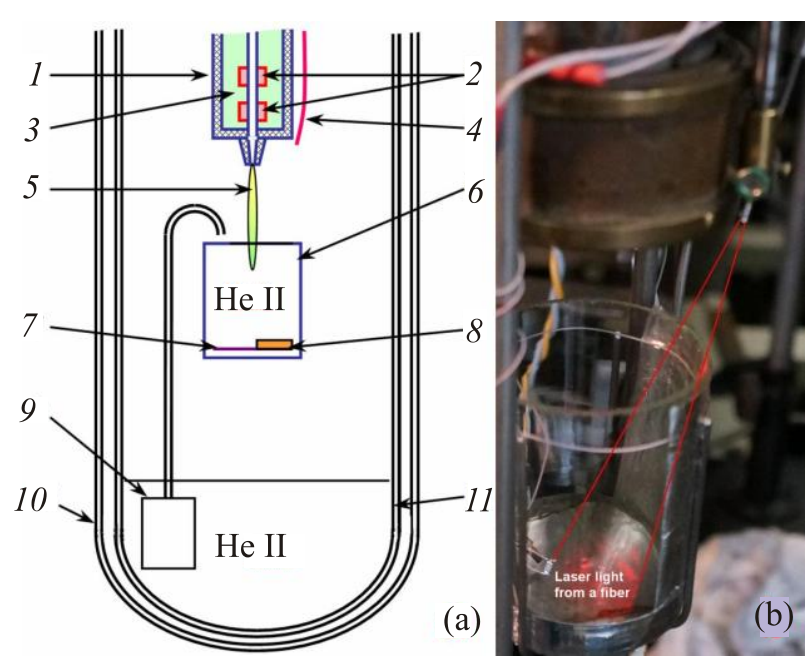

Fig. 1. (a) Scheme of the cryogenic part of the experimental setup: atom source (1), electrodes for an rf discharge (2), liquid nitrogen (3), optical fiber (4), gas jet (5), beaker for sample condensation (6), ion collector (7), thermometer (8), fountain pump in the main helium bath (9), nitrogen dewar (10), helium dewar (11); (b) Photo of the end of the atom source with beaker showing the position of the fiber collecting emission from IHC sample. Red laser beam illuminates the anode, showing the region of sample destruction.

steady state level of He II in the beaker (with the inner diameter of $40 \mathrm{~mm}$ ) was maintained by a fountain pump situated in the main liquid helium bath in the glass dewar. Calibrated Lake Shore thermometers were used for the temperature measurements. The pressure of the helium vapor in the dewar was measured with a RoseMount gauge S3051TA2 calibrated to work within the range 0-10 $\mathrm{kPa}$.

One setup modification has been done to extend the accessible spectral range to the UV range. The sample emission was directly collected by an optical fiber (pos. 4 in Fig. 1(a)) fixed above an IHC sample in the beaker, Fig. 1(b). The emission passed through fibers (inside and outside of the cryostat) and a vacuum feed-through to the AvaSpec-ULS2048XL-USB2 spectrometer. The spectrometer, the vacuum feed-through, and the fibers all together were calibrated for absolute intensity measurements by a light source AvaLight-DH-BAL-CAL. The spectrometer allowed us to detect luminescence within the spectral range from 200 to $1100 \mathrm{~nm}$ with resolution $\sim 2.5 \mathrm{~nm}$.

The ion currents accompanying the destruction of impurity-helium samples were collected by an electrode (a stainless steel disc, pos. 7 in Fig. 1(a)) connected directly to a picoammeter (Keithley 6485).

\section{Experimental results and discussion}

Firstly, we compare warm-ups of two samples prepared from a $\left[\mathrm{N}_{2}\right] /[\mathrm{Ar}] /[\mathrm{He}]=1 / 10 / 1000$ gas mixture (Fig. 2). The first sample has been condensed without action of an rf discharge, while the second one has been prepared from
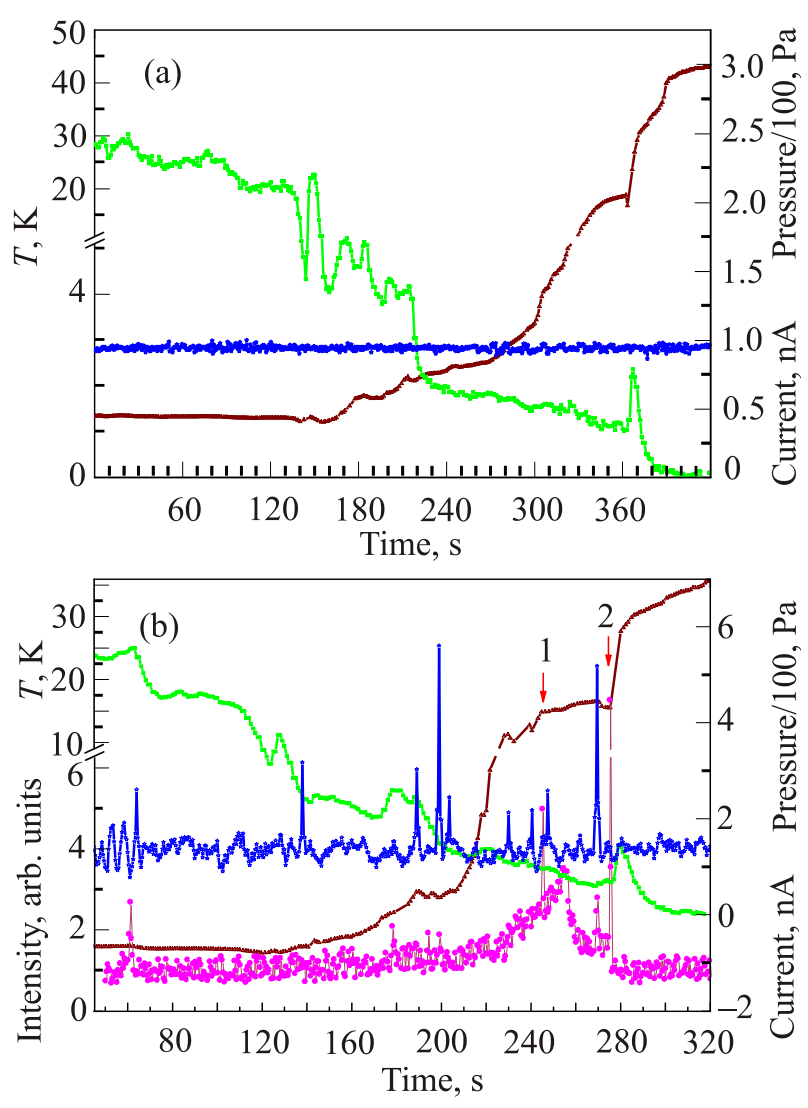

Fig. 2. (Color online) The temporal dependences of the temperature, pressure, current, and integrated luminescence intensity (brown, green, blue, and magenta lines, respectively) during destruction of sample prepared from a gas mixture $\left[\mathrm{N}_{2}\right] /[\mathrm{Ar}] /[\mathrm{He}]=$ $=1 / 10 / 1000$ : (a) the mixture condensed without action of the $\mathrm{rf}$ discharge; (b) the mixture passed through an rf discharge zone.

a gas mixture passed through the rf discharge zone. The sample destruction process was started after the onset of evaporation of liquid helium from the beaker. When there was no liquid helium in the bottom part of the dewar, the presence of liquid helium in the beaker could be monitored by the relation between the temperature measured in the beaker and the pressure detected in the helium dewar (the saturated helium vapor pressure). A characteristic feature of the IHC destruction process is a pressure peak corresponding to sublimation of helium atoms bound to the impurity nanocluster surfaces. These features can be seen for both samples in Fig. 2 (at $t=370$ and $280 \mathrm{~s}$, correspondingly), while the luminescence due to excited atomic and molecular species can be observed only from the IHCs prepared with an rf discharge. The luminescence intensity corresponds to the integrated (in the range from 220 to $1080 \mathrm{~nm}$ ) luminescence intensity.

The spectra of luminescence detected during the destruction of the second sample at times 1 and 2 (the points marked at Fig. 2(b)) are shown in Fig. 3. There are some differences in the spectra. The $\beta$-group (corresponding to the ${ }^{1} S_{-}{ }^{1} D$ transition of $\mathrm{O}$ atoms) and the Vegard-Kaplan 


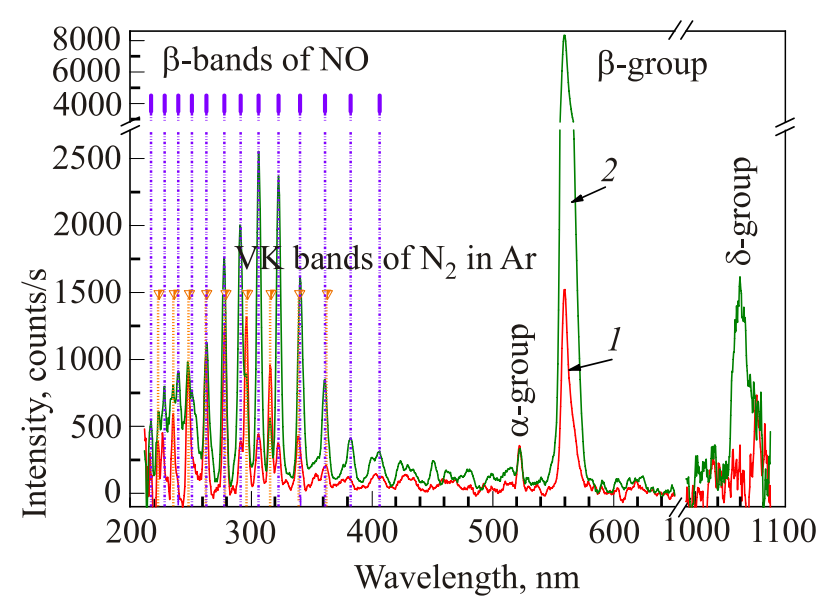

Fig. 3. (Color online) Luminescence spectra detected at the moments 1 and 2 (shown in Fig. 2(b)) during destruction of the sample prepared from a gas mixture $\left[\mathrm{N}_{2}\right] /[\mathrm{Ar}] /[\mathrm{He}]=1 / 10 / 1000$ passed through an rf discharge area.

(VK) bands of $\mathrm{N}_{2}$ dominate in the earlier spectrum, while the more intense $\beta$-group, and the $\beta$-system bands of NO molecules, together with the $\delta$-group of atomic nitrogen (corresponding to the transition ${ }^{2} P-{ }^{2} D$ ), dominate in the second spectrum detected during the final, brightest, flash. The relatively weak $\alpha$-group of atomic nitrogen, corresponding to the forbidden transition ${ }^{2} D-{ }^{4} S$, was present in both spectra. The dominance of emission due to species containing oxygen can be explained by a multishell structure of impurity clusters [13-16]: heavier particles (in this case $\mathrm{O}$ atoms) are involved in the reactions and the luminescence at the final stage of sample destruction when impurity clusters melt and fuse together.

The temporal dependences of the temperature, pressure, current, and integrated luminescence intensity during destruction of the sample prepared from a gas mixtures $\left[\mathrm{N}_{2}\right] /[\mathrm{Xe}] /[\mathrm{He}]=1 / 1 / 400$ are shown in Fig. 4(a). In this case the sample destruction process was started in He II as the liquid helium level decreased in the beaker. The orange vertical line marks the moment when liquid helium is gone from the beaker with the sample (He II had been pumped away from the main helium bath of the cryostat earlier). As one can see from Figs. 2 and 4(a), the destruction of impurity-helium condensates occurs through successive explosions of the "hottest" sample fragments, accompanied by peaks of temperature and pressure. After evaporation of LHe from the beaker, the sample temperature is kept stable due to evaporation of "weakly bound helium" - i.e., liquid helium contained in the sample pores [17]. At this stage, the sample annealing is accompanied by shrinking of the sample due to capillary forces. IHC samples shrink up to 10-fold [17], much more than highly porous aerogels (with a porosity of 95-99.5\%) [18,19], and become much denser (the impurity particle density increases up to $\sim 10^{21} \mathrm{~cm}^{-3}$ [20]). When the

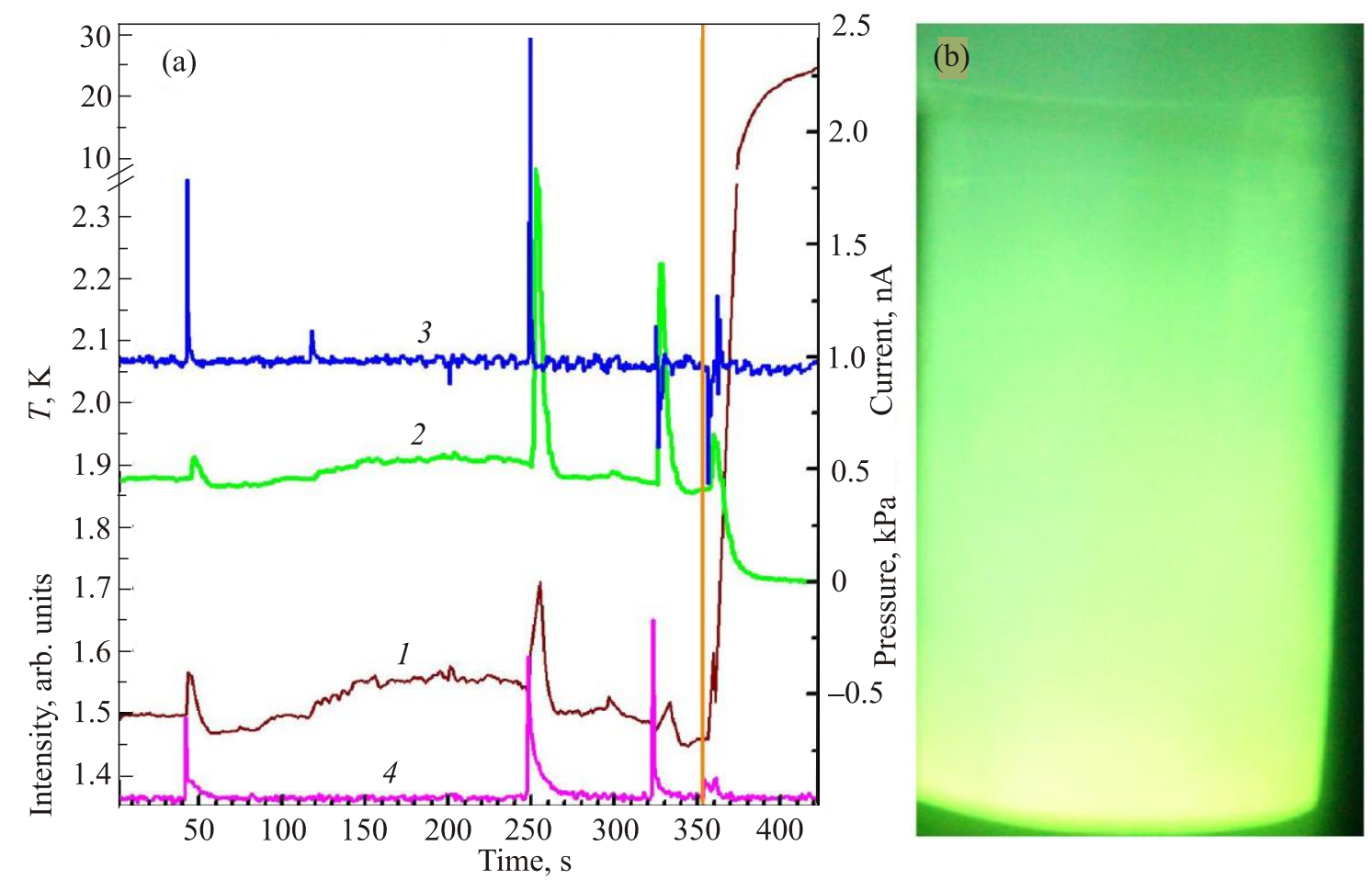

Fig. 4. (Color online) (a) The temporal dependences of the temperature (1), pressure (2), current (3), and integrated luminescence intensity (4) (brown, green, blue, and magenta line, respectively) during destruction of sample prepared from a gas mixture $\left[\mathrm{N}_{2}\right] /[\mathrm{Xe}] /[\mathrm{He}]=$ $=1 / 1 / 400$ passed through an $\mathrm{rf}$ discharge zone. The orange vertical line shows the time when liquid helium had completely evaporated from the beaker. (b) Photo of the third flash (at $t=323 \mathrm{~s}$ ). 


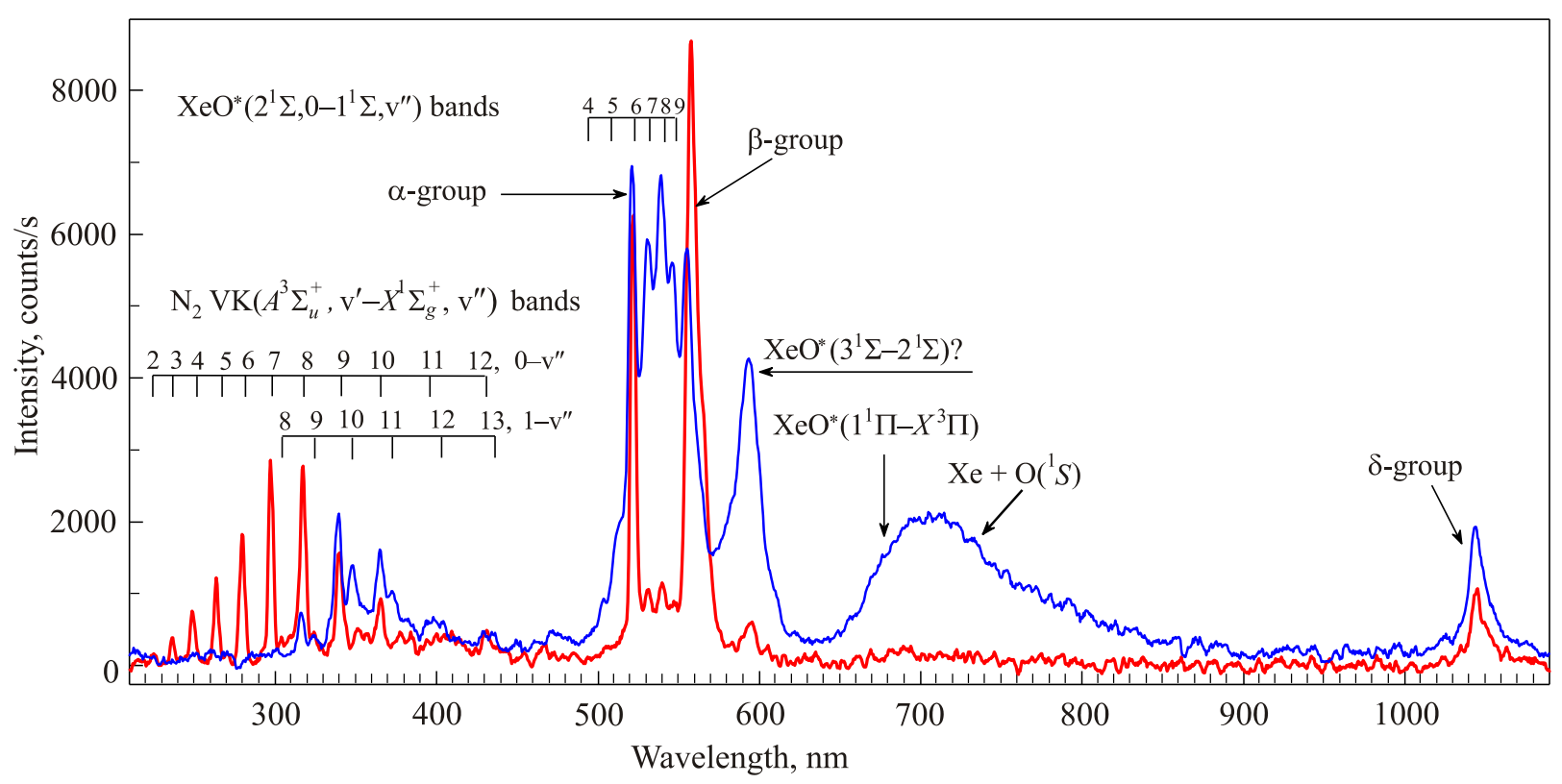

Puc. 5. (Color online) Luminescence spectra detected during destruction of samples prepared from gas mixtures $[\mathrm{Xe}] /\left[\mathrm{N}_{2}\right] /[\mathrm{He}]=$ $=1 / 1 / 400$ (red line) and $[\mathrm{Xe}] /\left[\mathrm{N}_{2}\right] /[\mathrm{He}]=1 / 10 / 2000$ (blue line).

sample become "dry" (no liquid helium in the pores remains), the beaker containing the sample starts to warm up because of heat transfer from the atom source. The warm-up causes sublimation of "strongly bound helium" - helium adsorbed on the impurity nanocluster surface [17,20] and coalescence of the nanoclusters into microcrystals [10,21]. The sublimation of helium shells, which had prevented recombination of radicals trapped on the impurity nanoclusters also initiates chemical reactions, and the formation of excited species responsible for optical emission during the sample destruction. The sublimation of helium atoms from the impurity nanocluster surface is revealed by the pressure peak, with a subsequent decrease of the pressure to zero (Figs. 2 and 4(a)).

A photo of the explosion (which corresponds to the flash \#3, at $t=323 \mathrm{~s}$ ) of the nitrogen-xenon-helium sample is shown in Fig. 4(b). As one can see the whole volume of the beaker is filled with glowing dust particles. The spectrum of this flash is shown in Fig. 5 (red line) together with the luminescence spectrum during destruction of the sample prepared from the gas mixture $[\mathrm{Xe}] /\left[\mathrm{N}_{2}\right] /[\mathrm{He}]=1 / 10 / 2000$ (blue line, the emission for wavelengths shorter than $310 \mathrm{~nm}$ is cut off due to the glass of the dewars). The intense Vegard-Kaplan bands, $\alpha-, \delta$-, and $\beta$-groups were observed along with the "green bands" and a band at $595 \mathrm{~nm}$, corresponding, possibly, to the cascade transitions of the $\mathrm{XeO}^{*}$ molecule, $\mathrm{XeO} *\left(2^{1} \sum-1^{1} \sum\right)$ and $\mathrm{Xe}^{+} \mathrm{O}^{-}\left(3^{1} \Sigma^{+}-2^{1} \Sigma\right)$ [22]. The band at $595 \mathrm{~nm}$ has never been observed from $\mathrm{XeO}^{*}$ in the gas phase, nor have the transitions from $\mathrm{XeO}\left(2^{1} \Sigma\right)$ in a xenon matrix [22,23]. We can see that the decrease of the xenon content in the condensed gas mixture is accompanied by a gain of the $\mathrm{XeO}^{*}$ molecule emission because single $\mathrm{Xe}$ and
$\mathrm{O}$ atoms reside over a larger volume (nitrogen matrix) with increase of nitrogen content. Therefore, for the first time the $\mathrm{XeO}^{*}$ spectra have been observed from molecules captured in $\mathrm{N}_{2}$ films surrounding the xenon cores of impurity nanoclusters.

The current pulses with amplitudes $\sim 1 \mathrm{nA}$ of positive and negative polarities were detected during destruction of the IHC samples under study. We suggest that electrons were carriers of negative charge, while the origin of positive carriers is still unclear. The ions can be formed (due to Penning ionization processes) and trapped into impurity nanoclusters growing in a gas jet [24]. Another possibility for ion formation is irradiation of impurity nanoclusters stabilized in bulk He II by VUV emission from the discharge area.

\section{Conclusions}

The destruction of impurity-helium condensates containing stabilized radicals is accompanied with pressure and luminescence peaks, and current pulses $(\sim \mathrm{nA})$.

The final pressure peak during sample destruction corresponds to sublimation of helium shells bound to the impurity nanocluster surface. The sublimation of helium shells which had prevented recombination of radicals trapped on the impurity nanoclusters initiates chemical reactions and the formation of excited species responsible for optical emission during the sample destruction.

The spectra observed during destruction of impurityhelium condensates containing stabilized radicals reveal that all of the emitting particles are localized within solid matrices. The spectral changes observed during the destructions of IHC samples confirm the multishell structure 
of impurity nanoclusters which form IHCs. The cluster growth occurs in the cold helium jet [11] when the heavier impurity particles form a cluster core which is later covered with lighter impurity particles.

The spectra of $\mathrm{XeO}^{*}$ molecules captured in $\mathrm{N}_{2}$ films have been observed for the first time.

\section{Acknowledgments}

This study was supported by the Program "Matter at High Energy Densities" of the Presidium of the Russian Academy of Sciences and by NSF grant No. DMR 1209255.

1. Formation and Trapping of Free Radicals, A.M. Bass and H.P. Broida (eds.), Academic Press (1960).

2. W.V. Bouldin and W. Gordy, Phys. Rev. 135, A 806 (1964).

3. V.E. Bondybey, M. Rasanen, and A. Lammers, Annu. Rep. Prog. Chem., Sect. C 95, 331 (1999).

4. L. Andrews, Ann. Rev. Phys. Chem. 30, 79 (1979).

5. L.B. Knight, Acc. Chem. Res. 19, 313 (1986).

6. M.E. Jacox and W.E. Thompson, Research on Chemical Intermediates 12, 33 (1989).

7. A.N. Ogurtsov, E.V. Savchenko, M. Kirm, B. Steeg, and G. Zimmerer, J. Electron Spectrosc. Relat. Phenom. 101, 479 (1999).

8. R.E. Boltnev, I.B. Bykhalo, I.N. Krushinskaya, A.A. Pelmenev, V.V. Khmelenko, D.M. Lee, I.V. Khyzhniy, S.A. Uyutnov, E.V. Savchenko, A.N. Ponomaryov, G.B. Gumenchuk, and V.E. Bondybey, Fiz. Nizk. Temp. 39, 580 (2013) [Low Temp. Phys. 39, 451 (2013)].

9. E.B. Gordon, L.P. Mezhov-Deglin, and O.F. Pugachev, JETP Lett. 19, 103 (1974).

10. V. Kiryukhin, B. Keimer, R.E. Boltnev, V.V. Khmelenko, and E.B. Gordon, Phys. Rev. Lett. 79, 1774 (1997).

11. V. Kiryukhin, E.P. Bernard, V.V. Khmelenko, R.E. Boltnev, N.V. Krainyukova, and D.M. Lee, Phys. Rev. Lett. 98, 195506 (2007).
12. S.I. Kiselev, V.V. Khmelenko, D.M. Lee, V. Kiryukhin, R.E. Boltnev, E.B. Gordon, and B. Keimer, Phys. Rev. B 65, 024517 (2001).

13. O.G. Danylchenko, Yu.S. Doronin, S.I. Kovalenko, and V.N. Samovarov, JETP Lett. 84, 324 (2006).

14. T. Laarmann, H. Wabnitz, K. von Haeften, and T. Möller, J. Chem. Phys. 128, 014502 (2008).

15. R.E. Boltnev, V.V. Khmelenko, and D.M. Lee, Fiz. Nizk. Temp. 36, 484 (2010) [Low Temp. Phys. 36, 382 (2010)].

16. V.V. Khmelenko, A.A. Pelmenev, I.N. Krushinskaya, I.B. Bykhalo, R.E. Boltnev, and D.M. Lee, J. Low Temp. Phys. 171, 302 (2013).

17. R.E. Boltnev, E.B. Gordon, I.N. Krushinskaya, M.V. Martynenko, A.A. Pelmenev, E.A. Popov, V.V. Khmelenko, and A.F. Shestakov, Fiz. Nizk. Temp. 23, 753 (1997) [Low Temp. Phys. 23, 567 (1997)].

18. T. Herman, J. Day, and J. Beamish, Phys. Rev B 73, 094127 (2006)

19. H. Kato, W. Miyashita, R. Nomura, and Y. Okuda, J. Low Temp. Phys. 148, 621 (2007).

20. E.P. Bernard, R.E. Boltnev, V.V. Khmelenko, V. Kiryukhin, S.I. Kiselev, and D.M. Lee, J. Low Temp. Phys. 134, 169 (2004).

21. N.V. Krainyukova, R.E. Boltnev, E.P. Bernard, V.V. Khmelenko, D.M. Lee, and V. Kiryukhin, Phys. Rev. Lett. 109, 245505 (2012).

22. A.G. Belov and E.M. Yurtaeva, Fiz. Nizk. Temp. 27, 1268 (2001) [Low Temp. Phys. 27, 938 (2001)].

23. W.G. Lawrence and V.A. Apkarian, J. Chem. Phys. 97, 2229 (1992).

24. A.A. Pelmenev, I.N. Krushinskaya, I.B. Bykhalo, and R.E. Boltnev, On Charged Impurity Structures in Liquid Helium, to be published. 